\title{
DIAGNOSE NUTRICIONAL PELOS MÉTODOS DRIS E FAIXAS DE CONCENTRAÇÃO PARA ALGODOEIRO CULTIVADO SOB CERRADO ${ }^{1}$
}

\author{
Roberta de Freitas Souza ${ }^{2}$, Wilson Mozena Leandro ${ }^{3}$, \\ Noé Barroso da Silva ${ }^{4}$, Paulo César Ribeiro da Cunha ${ }^{5}$, Paulo Alcanfor Ximenes ${ }^{3}$
}

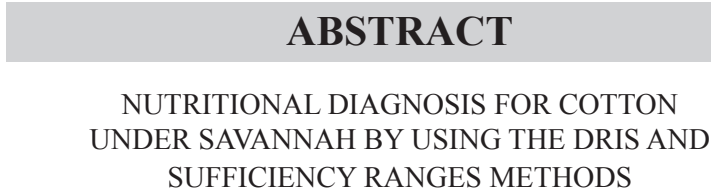

Brazil is the fourth largest cotton exporter and fifth largest producer in the world, however, it is predominantly cultivated under savannah soils, which present natural nutrients limitations. The objective of this study was to diagnose the nutritional factors that most limit high cotton yields, by using leaf analysis interpreted with the aid of the DRIS and sufficiency ranges techniques. So, forty-eight leaf samples were collected in a commercial area of Silvânia, Goiás State, Brazil. Ten cotton leaves were collected to a compound sample, at the full flowering stage (90 day after seeding). For interpreting the leaf analyses results, by using the DRIS technique, indexes for each nutrient from each sample were calculated, according to Alvarez \& Leite (1992). For the sufficiency range interpretation, standard values were used. Both methods evaluated N, S, B, and $\mathrm{Zn}$ as the most limiting nutrients. The DRIS method showed higher sensibility for diagnosing nutritional deficiencies, especially for the B and Zn micronutrients.

KEY-WORDS: Gossypium sp.; leaf analysis; DRIS index; nutritional status.

\section{INTRODUÇÃO}

O Brasil é o quarto maior exportador e o quinto maior produtor mundial de fibras de algodão, tendo produzido 1.194,5 mil toneladas de plumas, na safra 2009/2010. O algodão brasileiro é reconhecido por sua excelência, sendo exportado para mercados exigentes, como a Coreia do Sul, Turquia e Indonésia (Anuário... 2010).

A área plantada, na safra 2010/2011, foi de $1.034,7$ mil hectares, o que corresponde a um aumento de $56,1 \%$, quando comparada à safra 2009/2010,

\section{RESUMO}

O Brasil é o quarto maior exportador e o quinto maior produtor mundial de algodão. Entretanto, a cultura é produzida, predominantemente, em áreas sob solos de Cerrado, que apresentam limitações naturais de nutrientes. O objetivo do trabalho foi diagnosticar os fatores nutricionais mais limitantes à obtenção de altas produtividades, para a cultura do algodão, por meio de análises foliares interpretadas pelos métodos DRIS e Faixas de Concentração. Foram coletadas 48 amostras de folhas, em área comercial do município de Silvânia (GO). Coletaram-se 10 folhas do algodoeiro, para uma amostra composta, na época de florescimento pleno (90 dias após o plantio). Na interpretação dos resultados das análises foliares pelo método DRIS, foram calculados índices para cada nutriente de cada amostra, pelo procedimento de Alvarez \& Leite (1992). Na interpretação pelas Faixas de Concentração, utilizaram-se padrões tabelados. Os dois métodos avaliaram, como nutrientes mais limitantes, $\mathrm{N}, \mathrm{S}, \mathrm{B}$ e Zn. O método DRIS apresentou maior sensibilidade para diagnosticar problemas nutricionais, especialmente para os micronutrientes $\mathrm{B}$ e $\mathrm{Zn}$.

PALAVRAS-CHAVE: Gossypium sp.; análise foliar; índice DRIS; estado nutricional.

com uma produção de 4.990,3 mil toneladas de algodão em caroço, 64,3\% superior à da safra anterior (Conab 2011). Este cenário é justificado pelo recorde histórico da alta de preços, provocado pela forte redução dos estoques mundiais, ocasionada pela elevada demanda, principalmente por parte da indústria têxtil asiática.

Atualmente, a região Centro-Oeste responde por $63,4 \%$ do algodão produzido no Brasil. Somando-se a produção do Centro-Oeste com a da Bahia e do Maranhão, o algodão do Cerrado representa mais de 95,0\% da produção nacional (Conab 2011).

1. Trabalho recebido em set./2009 e aceito para publicação em maio/2011 (n registro: PAT 7461/ DOI: 10.5216/pat.v41i2.7461).

2. Instituto Federal de Educação, Ciência e Tecnologia do Tocantins, Campus Araguatins, Araguatins, TO, Brasil. E-mail: robertadfs@yahoo.com.br.

3. Universidade Federal de Goiás, Escola de Agronomia e Engenharia de Alimentos, Setor de Solos, Goiânia, GO, Brasil. E-mails: wilson-ufg@bol.com.br, ximenes@agro.ufg.br.

4. Embrapa Arroz e Feijão, Setor de Solos, Santo Antônio de Goiás, GO, Brasil. E-mail: noebarroso@cnpaf.embrapa.br. 5. Instituto Federal de Educação, Ciência e Tecnologia Goiano, Campus Urutaí, Urutaí, GO, Brasil. E-mail: pcdacunha@hotmail.com. 
A região do Cerrado é privilegiada, por possuir características excelentes para o cultivo do algodoeiro, como a topografia plana, que facilita a mecanização, e condições climáticas adequadas, entretanto, os solos da região apresentam limitações químicas, quanto ao cultivo, devido à sua baixa fertilidade natural (Lopes 1984). Para que o algodoeiro expresse todo o seu potencial produtivo, são necessários adequados programas de correção da acidez do solo e adubação. A primeira etapa, na implantação destes programas, é a diagnose do estado nutricional da cultura. A análise foliar é uma ferramenta eficiente neste diagnóstico, pois a planta é o próprio extrator de nutrientes do solo, possibilitando um diagnóstico nutricional direto e preciso (Beaufils 1973). Os métodos mais empregados na interpretação dos resultados das análises foliares são: Faixas de Concentração (FC) e Sistema de Diagnose e Recomendação (DRIS).

O método FC compara, individualmente, os níveis de cada nutriente, com parâmetros previamente tabelados (Chapmann 1973, Walsh \& Beaton 1973). Esta técnica de interpretação apresenta desvantagens, pois não leva em consideração a interação existente entre nutrientes e a variação de suas concentrações, com o desenvolvimento da planta (Sumner 1977).

O DRIS, preconizado por Beaufils (1973), incorpora o conceito de equilíbrio entre minerais, nos tecidos das plantas (Baldock \& Schulte 1996). A técnica se baseia no cálculo de índices para cada nutriente, avaliados em função da relação das razões das concentrações de cada elemento com os demais, comparando-os, dois a dois, com outras relações consideradas padrões, obtidas de uma população de plantas altamente produtivas.

A particularização desta população de plan-tas, restringindo a aplicação do DRIS a uma determinada região, material genético e condições edafoclimáticas, é uma medida de refinamento importante, para melhorar a precisão do diagnóstico (Dara et al.1992, Reis Júnior \& Monnerat 2003). O potencial de utilização deste sistema foi demonstrado em vários trabalhos realizados com algodão (Silva et al. 2007, Kurihara et al. 2008, Morais 2008, Valdameri \& Pouyú-Rojas 2008, Souza 2009, Serra et al. 2010a).

$\mathrm{Na}$ interpretação dos índices DRIS, o índice negativo indica que o nutriente está abaixo do nível ótimo, enquanto o índice positivo indica que o nutriente está acima do nível ótimo. Se o índice DRIS de um nutriente é igual a zero, este elemento está em equilíbrio com os outros nutrientes (Beaufils 1973). O somatório dos valores absolutos dos índices DRIS fornece o índice de balanço nutricional (IBN), que expressa o equilíbrio nutricional da cultura amostrada. Quanto menor o IBN, mais próxima a amostra estará do equilíbrio nutricional (Beaufils 1973, Walworth \& Sumner 1987).

O objetivo deste trabalho foi diagnosticar os fatores nutricionais mais limitantes à obtenção de altas produtividades, para a cultura do algodão, pela análise foliar, interpretada pelos métodos DRIS e Faixas de Concentração, em uma lavoura comercial no município de Silvânia (GO).

\section{MATERIAL E MÉTODOS}

O trabalho foi desenvolvido de dezembro de 2007 a abril de 2008, na Fazenda Cedro, localizada no município de Silvânia (16²9'21'’S e 48²2'32”W), sudeste de Goiás, com a cultura do algodoeiro, cultivares Fibermax 966 e 993. Os solos predominantes nesta região são do tipo Latossolo Vermelho (Embrapa 1999). A região possui clima Aw, segundo a classificação de Köppen, caracterizado pela existência de duas estações bem definidas: uma chuvosa e quente, que se prolonga de outubro a abril, e outra fria e seca, de maio a setembro.

De acordo com o levantamento realizado na propriedade, a cultura do algodão foi implantada dentro de um sistema de cultivo rotacionado com soja e milheto, sob plantio semidireto. Na safra 2006/2007, foi semeada a cultura da soja e, após a colheita, semeou-se milheto. Na safra de realização deste estudo (2007/2008), o plantio do algodão foi efetuado entre os dias 7 e 9 de dezembro de 2007. A densidade populacional foi de 133.333 mil plantas por hectare, sendo o espaçamento de $0,90 \mathrm{~m}$ entre as linhas de plantio.

Segundo este levantamento, para correção e adubação do solo, os agricultores efetuaram calagem com calcário dolomítico, na dosagem de $1,50 \mathrm{Mg} \mathrm{ha}^{-1}$ de calcário, sendo utilizados, no plantio, $400 \mathrm{~kg} \mathrm{ha}^{-1}$ da formulação 08-30-10, e, em cobertura, aplicados a lanço, $250 \mathrm{~kg} \mathrm{ha}^{-1}$ de ureia da dose no estádio B2 e metade em F2 e $150 \mathrm{~kg} \mathrm{ha}^{-1}$ de $\mathrm{KCl}$ (estádio B2). Foram realizadas, também, algumas adubações foliares com produtos à base de fósforo, magnésio, cálcio, boro e manganês, conforme experiência local dos agricultores. 
Para diagnosticar o estado nutricional da cultura, foram coletadas 48 amostras compostas, distribuídas, de forma representativa, na área de um talhão de, aproximadamente, 92 ha. Coletou-se a $5^{\text {a }}$ folha a partir da ponta da haste principal da planta de algodão, na época de florescimento pleno, aos 95 dias após o plantio (Malavolta et al. 1997). Foram coletadas dez folhas (uma de cada planta), para constituir uma amostra composta.

Cada amostra composta foi embalada em saco de papel e etiquetada. O material, depois de lavado com água destilada, foi colocado para secar em uma estufa de circulação forçada, a $65^{\circ} \mathrm{C}$, e, em seguida, triturado em moinho de aço do tipo Willey. Este material foi digerido pela mistura digestora nitro-perclórica (2:1), para a obtenção de um extrato, no qual foram determinadas as concentrações foliares totais de fósforo $(\mathrm{P})$, potássio $(\mathrm{K})$, cálcio $(\mathrm{Ca})$, magnésio $(\mathrm{Mg})$, enxofre $(\mathrm{S})$, cobre $(\mathrm{Cu})$, manganês $(\mathrm{Mn})$, Ferro (Fe), zinco (Zn) e boro (B), segundo metodologia descrita por Bataglia et al. (1978). Para a determinação das concentrações foliares totais de nitrogênio $(\mathrm{N})$, foi empregada a digestão sulfúrica e a metodologia descrita por Bataglia et al. (1978).

Nos 48 resultados de análises foliares, foram aplicados testes de estatística univariada, sendo calculadas as médias, coeficientes de variação e valores máximos e mínimos, e, para avaliar a normalidade da população, aplicou-se o teste de Shapiro-Wilk. O programa estatístico utilizado foi o Statistical Analysis System - SAS (Freund \& Littell 1981). Para interpretação da análise foliar, foi empregada a concentração de nutrientes considerados adequados para o algodoeiro (Tabela 1), descritos por Malavolta et al. (1997).

$\mathrm{Na}$ interpretação pelo método DRIS, calcularam-se os índices DRIS, utilizando-se todas as relações entre as concentrações dos nutrientes $\mathrm{N}, \mathrm{P}$, $\mathrm{K}, \mathrm{Ca}, \mathrm{Mg}, \mathrm{S}, \mathrm{B}, \mathrm{Cu}, \mathrm{Fe}, \mathrm{Mn}$ e Zn obtidas pela análise foliar, para todas as 48 amostras. Os índices foram calculados adotando-se, como padrão, a população de referência com produtividade maior que $4.000 \mathrm{~kg} \mathrm{ha}^{-1}$ de algodão em caroço, do banco de dados do Setor de Solos da Escola de Agronomia e Engenharia de Alimentos da Universidade Federal de Goiás (Morais 2008, Souza 2009).

O procedimento utilizado para o cálculo dos índices DRIS foi proposto por Alvarez \& Leite (1992), como se segue: Índice $A=Z(A / B)+$ $\mathrm{Z}(\mathrm{A} / \mathrm{C})+\ldots+\mathrm{Z}(\mathrm{A} / \mathrm{N})-\mathrm{Z}(\mathrm{B} / \mathrm{A})-\mathrm{Z}(\mathrm{C} / \mathrm{A})-\ldots . . \mathrm{Z}$
Tabela 1. Concentração de nutrientes foliares considerados adequados (faixas de concentração) na matéria seca de folhas de algodoeiro, no período de florescimento, para interpretação de análise foliar.

\begin{tabular}{llrl}
\hline \multirow{2}{*}{ Nutrientes } & \multicolumn{3}{c}{ Critério de interpretação } \\
\cline { 2 - 4 } & \multicolumn{1}{c}{ Baixo } & Adequado & Alto \\
\hline $\mathrm{N}\left(\mathrm{g} \mathrm{kg}^{-1}\right)$ & $<45,0$ & $45,0-50,0$ & $>50,0$ \\
$\mathrm{P}\left(\mathrm{g} \mathrm{kg}^{-1}\right)$ & $<2,5$ & $2,5-4,0$ & $>4,0$ \\
$\mathrm{~K}\left(\mathrm{~g} \mathrm{~kg}^{-1}\right)$ & $<21,0$ & $21,0-24,0$ & $>24,0$ \\
$\mathrm{Ca}\left(\mathrm{g} \mathrm{kg}^{-1}\right)$ & $<30,0$ & $30,0-35,0$ & $>35,0$ \\
$\mathrm{Mg}\left(\mathrm{g} \mathrm{kg}^{-1}\right)$ & $<4,0$ & $4,0-5,0$ & $>5,0$ \\
$\mathrm{~S}\left(\mathrm{~g} \mathrm{~kg}^{-1}\right)$ & $<5,0$ & $5,0-6,0$ & $>6,0$ \\
$\mathrm{~B}\left(\mathrm{mg} \mathrm{kg}^{-1}\right)$ & $<40,0$ & $40,0-50,0$ & $>50,0$ \\
$\mathrm{Cu}\left(\mathrm{mg} \mathrm{kg}^{-1}\right)$ & $<8,0$ & $8,0-10,0$ & $>10,0$ \\
$\mathrm{Fe}\left(\mathrm{mg} \mathrm{kg}^{-1}\right)$ & $<100,0$ & $100,0-150,0$ & $>150,0$ \\
$\mathrm{Mn}\left(\mathrm{mg} \mathrm{kg}^{-1}\right)$ & $<60,0$ & $60,0-70,0$ & $>70,0$ \\
$\mathrm{Zn}\left(\mathrm{mg} \mathrm{kg}^{-1}\right)$ & $<25,0$ & $25,0-65,0$ & $>65,0$ \\
\hline
\end{tabular}

Fonte: Malavolta (2006).

(N/A)/ 2 (n - 1), em que Z(A/B) até Z(N/A) são as relações normais reduzidas diretas e inversas das concentrações de todos os nutrientes, em relação ao nutriente $\mathrm{A}$, determinadas pela análise foliar, e $\mathrm{n}-1$ o número de relações possíveis.

As relações normais reduzidas foram calculadas pelo procedimento de Beaufils (1973), como se segue: se $A / B>a / b, Z(A / B)=[(A / B) /(a / b)-1]^{*}[100 /$ $\mathrm{CV}]$; se $\mathrm{a} / \mathrm{b}>\mathrm{A} / \mathrm{B}, \mathrm{Z}(\mathrm{A} / \mathrm{B})=[1-(\mathrm{a} / \mathrm{b}) /(\mathrm{A} / \mathrm{B})-1]^{*}[100 /$ $\mathrm{CV}]$, em que $\mathrm{A} / \mathrm{B}$ é o quociente das concentrações dos nutrientes A e B da amostra em análise e interpretação; a/b a média da razão dos nutrientes A e B da população padrão; e CV o coeficiente de variação da razão dos nutrientes $\mathrm{A}$ e $\mathrm{B}$ da população padrão, que satisfaz definido nível mínimo de produtividade.

Para interpretação dos índices, foi empregado o procedimento padrão proposto por Beaufils (1973). Além do procedimento padrão, também foi feita interpretação baseada no procedimento proposto por Leandro (1998). Para este autor, a interpretação é feita considerando-se a percentagem de ocorrência de limitação total, avaliando-se todas as variáveis com índices negativos de cada amostra. A percentagem de ocorrência de limitação total, neste trabalho, seria equivalente à classe baixa das faixas de concentração. Obteve-se, também, a percentagem de ocorrência na primeira, segunda e terceira ordem, que correspondem ao primeiro, segundo, e terceiro índice mais negativo, respectivamente, em cada amostra. Calculou-se o IBN, segundo Beaufils (1973), pela soma, em módulo, dos índices DRIS. 


\section{RESULTADOS E DISCUSSÃO}

Observa-se que os valores médios obtidos de $\mathrm{K}\left(15,46 \mathrm{~g} \mathrm{~kg}^{-1}\right), \mathrm{S}\left(2,41 \mathrm{~g} \mathrm{~kg}^{-1}\right)$ e Zn $\left(18,73 \mathrm{~g} \mathrm{~kg}^{-1}\right)$ (Tabela 2) estão muito abaixo dos níveis adequados propostos por Malavolta (2006) (Tabela 1). Já os valores médios de $\mathrm{Fe}\left(340,85 \mathrm{mg} \mathrm{kg}^{-1}\right)$ e $\mathrm{Mn}$ $\left(158,47 \mathrm{mg} \mathrm{kg}^{-1}\right)$ estão muito acima destes níveis (Tabela 2). Apenas as concentrações médias de $\mathrm{Ca}$ e B enquadraram-se nas faixas adequadas, enquanto os valores médios dos nutrientes $\mathrm{N}, \mathrm{P}, \mathrm{Mg}$ e $\mathrm{Cu}$, apesar de não estarem dentro das faixas adequadas, apresentaram valores próximos (Tabela 2).

Os maiores coeficientes de variação foram obtidos para as concentrações de $\mathrm{Mg}(19,32 \%), \mathrm{B}$ $(39,14 \%), \mathrm{Fe}(27,67 \%)$ e $\mathrm{Mn}(25,02 \%)$. Os altos valores de coeficientes de variação são justificados por se tratar de uma propriedade de solos que apresentam parâmetros de fertilidade variados. Os testes de Shapiro-Wilk, com exceção do teor de N, P, K, Ca e Fe, foram significativos para todas as variáveis, indicando que existem desvios da distribuição normal. Porém, decidiu-se não transformar os dados.

Quanto à distribuição de frequência, para as variáveis da análise foliar (Tabela 3), os nutrientes que apresentaram maiores percentagens de amostras abaixo dos níveis adequados, segundo Malavolta (2006), foram o S (100\%), K (97,9\%), Zn (97,9\%), B $(66,7 \%)$ e $\mathrm{N}(62,5 \%)$, enquanto os nutrientes que apresentaram maiores percentagens de amostras acima dos níveis adequados foram o $\mathrm{Fe}(100 \%), \mathrm{P}(95,8 \%)$, $\mathrm{Cu}(95,8 \%), \operatorname{Mn}(95,8 \%), \operatorname{Mg}(60,4 \%)$ e B $(33,3 \%)$.

Tabela 2. Valores máximos, mínimos, médias, coeficientes de variação $(\mathrm{CV})$ e teste $\mathrm{W}$, para os nutrientes $\mathrm{N}, \mathrm{P}, \mathrm{K}$, $\mathrm{Ca}, \mathrm{Mg}, \mathrm{S}, \mathrm{B}, \mathrm{Cu}, \mathrm{Fe}, \mathrm{Mn}$ e $\mathrm{Zn}$, obtidos pela análise foliar de 48 amostras de folhas de algodoeiro (Silvânia, GO, safra 2007/2008).

\begin{tabular}{lrrrrl}
\hline Variável & Mínimo Máximo & Média & $\mathrm{CV}(\%)$ & Teste W \\
\hline $\mathrm{N}\left(\mathrm{g} \mathrm{kg}^{-1}\right)$ & 36,12 & 50,68 & 42,94 & 8,66 & $0,97^{\text {ns }}$ \\
$\mathrm{P}\left(\mathrm{g} \mathrm{kg}^{-1}\right)$ & 3,54 & 5,62 & 4,71 & 10,45 & $0,95^{\text {ns }}$ \\
$\mathrm{K}\left(\mathrm{g} \mathrm{kg}^{-1}\right)$ & 11,00 & 21,40 & 15,46 & 14,39 & $0,96^{\text {ns }}$ \\
$\mathrm{Ca}\left(\mathrm{g} \mathrm{kg}^{-1}\right)$ & 25,00 & 40,00 & 31,91 & 11,46 & $0,97^{\text {ns }}$ \\
$\mathrm{Mg}\left(\mathrm{g} \mathrm{kg}^{-1}\right)$ & 3,00 & 8,00 & 5,77 & 19,32 & $0,90^{* *}$ \\
$\mathrm{~S}\left(\mathrm{~g} \mathrm{~kg}^{-1}\right)$ & 1,25 & 2,95 & 2,41 & 11,87 & $0,89^{* *}$ \\
$\mathrm{~B}\left(\mathrm{mg} \mathrm{kg}^{-1}\right)$ & 36,16 & 109,59 & 49,10 & 39,14 & $0,63^{* *}$ \\
$\mathrm{Cu}\left(\mathrm{mg} \mathrm{kg}^{-1}\right)$ & 10,00 & 17,00 & 13,87 & 13,67 & $0,90^{* *}$ \\
$\mathrm{Fe}\left(\mathrm{mg} \mathrm{kg}^{-1}\right)$ & 164,00 & 608,00 & 340,85 & 27,67 & $0,97^{\text {ns }}$ \\
$\mathrm{Mn}\left(\mathrm{mg} \mathrm{kg}^{-1}\right)$ & 48,00 & 275,00 & 158,47 & 25,02 & $0,94^{*}$ \\
$\mathrm{Zn}\left(\mathrm{mg} \mathrm{kg}^{-1}\right)$ & 14,70 & 26,40 & 18,73 & 12,74 & $0,95^{*}$ \\
\hline${ }^{1}$ Teste de Shapiro-Wilk. * Significativo a $5 \% . * *$ Significativo a $1 \%$. ${ }^{\text {ns }}$ Não \\
significativo.
\end{tabular}

Tabela 3. Distribuição de frequência dos nutrientes N, P, K, Ca, $\mathrm{Mg}, \mathrm{S}, \mathrm{B}, \mathrm{Cu}, \mathrm{Fe}, \mathrm{Mn}$ e $\mathrm{Zn}$, obtida pela análise foliar de 48 amostras de folhas de algodoeiro (Silvânia, GO, safra 2007/2008).

\begin{tabular}{|c|c|c|c|}
\hline \multirow{3}{*}{ Variável } & \multicolumn{3}{|c|}{ Critério de interpretação ${ }^{1}$} \\
\hline & Abaixo & Adequado & Acima \\
\hline & \multicolumn{3}{|c|}{$\%$} \\
\hline $\mathrm{N}\left(\mathrm{g} \mathrm{kg}^{-1}\right)$ & 62,5 & 33,3 & 4,2 \\
\hline$P\left(\mathrm{~g} \mathrm{~kg}^{-1}\right)$ & 0,0 & 4,2 & 95,8 \\
\hline $\mathrm{K}\left(\mathrm{g} \mathrm{kg}^{-1}\right)$ & 97,9 & 2,1 & 0,0 \\
\hline $\mathrm{Ca}\left(\mathrm{g} \mathrm{kg}^{-1}\right)$ & 29,2 & 52,1 & 18,7 \\
\hline $\operatorname{Mg}\left(\mathrm{g} \mathrm{kg}^{-1}\right)$ & 4,2 & 35,4 & 60,4 \\
\hline $\mathrm{S}\left(\mathrm{g} \mathrm{kg}^{-1}\right)$ & 100,0 & 0,0 & 0,0 \\
\hline $\mathrm{B}\left(\mathrm{mg} \mathrm{kg}^{-1}\right)$ & 66,7 & 0,0 & 33,3 \\
\hline $\mathrm{Cu}\left(\mathrm{mg} \mathrm{kg}{ }^{-1}\right)$ & 0,0 & 4,2 & 95,8 \\
\hline $\mathrm{Fe}\left(\mathrm{mg} \mathrm{kg}^{-1}\right)$ & 0,0 & 0,0 & 100,0 \\
\hline $\operatorname{Mn}\left(\mathrm{mg} \mathrm{kg}^{-1}\right)$ & 4,2 & 0,0 & 95,8 \\
\hline $\mathrm{Zn}\left(\mathrm{mg} \mathrm{kg}^{-1}\right)$ & 97,9 & 2,1 & 0,0 \\
\hline
\end{tabular}

${ }^{1}$ Baseado nos níveis adequados descritos por Malavolta (2006).

As faixas de interpretação propostas para micronutrientes, por Malavolta (2006), são muito estreitas e acabam enquadrando a maioria das amostras na classe excessiva, principalmente para $\mathrm{Mn}$, $\mathrm{Cu}$ e Fe. Silva et al. (1995) apresentam, para estes micronutrientes, faixas com amplitudes bem maiores.

De acordo com as faixas de concentração propostas por Malavolta (2006), a ordem de limitação por deficiência dos nutrientes foi $\mathrm{S}>\mathrm{Zn}=\mathrm{K}>\mathrm{B}>$ $\mathrm{N}>\mathrm{Ca}>\mathrm{Mg}>\mathrm{Mn}$, enquanto, por excesso, foi $\mathrm{Fe}>$ $\mathrm{Mn}=\mathrm{P}=\mathrm{Cu}>\mathrm{Mg}>\mathrm{B}>\mathrm{Ca}>\mathrm{N}$ (Tabela 3).

$\mathrm{Na}$ Tabela 4, estão apresentados os índices DRIS, para cada nutriente de cada amostra, a ordem de limitação dos nutrientes e o IBN. A partir da ordenação dos índices, foi possível obter uma distribuição de frequências dos índices (Tabela 5). Esta distribuição de frequência, ao contrário da obtida para as amostras interpretadas pelas Faixas de Concentração, não possibilita uma interpretação em classes definidas (baixa, média, adequada, alta, etc.). Assim, os dados foram interpretados segundo o procedimento de Leandro (1998), considerando-se a percentagem de ocorrência de limitação total e em primeira, segunda e terceira ordem de limitação.

$\mathrm{Na}$ interpretação da percentagem de ocorrência de limitação total, em algumas situações, são consideradas deficientes as variáveis negativas muito próximas ao estado de equilíbrio nutricional (índices iguais a zero), o que pode provocar distorções nas interpretações (Leandro 1998). Devido a este problema, consideraram-se, na interpretação, as três primeiras 
Tabela 4. Índices DRIS, calculados pelo procedimento de Alvarez \& Leite (1992), e Índice de Balanço Nutricional (IBN) obtidos pelas análises foliares de 48 amostras de folhas de algodoeiro (Silvânia, GO, safra 2007/2008).

\begin{tabular}{|c|c|c|c|c|c|c|c|c|c|c|c|c|c|}
\hline \multirow{2}{*}{$\mathrm{A}^{1}$} & \multicolumn{11}{|c|}{ Índices } & \multirow{2}{*}{$\mathrm{IBN}^{2}$} & \multirow{2}{*}{$\begin{array}{c}\text { Ordem de limitação } \\
\text { (deficiência a excesso) }\end{array}$} \\
\hline & $\mathrm{N}$ & $\mathrm{P}$ & K & $\mathrm{Ca}$ & $\mathrm{Mg}$ & $\mathrm{S}$ & $\mathrm{B}$ & $\mathrm{Cu}$ & $\mathrm{Fe}$ & $\mathrm{Mn}$ & $\mathrm{Zn}$ & & \\
\hline 1 & 2 & 5 & 16 & 0 & 5 & -5 & -22 & 9 & -13 & 2 & -3 & 84 & $\mathrm{~B}>\mathrm{Fe}>\mathrm{S}>\mathrm{Zn}>\mathrm{Ca}>\mathrm{N}=\mathrm{Mn}>\mathrm{P}=\mathrm{Mg}>\mathrm{Cu}>\mathrm{K}$ \\
\hline 2 & -4 & 1 & 14 & 3 & 7 & -1 & -25 & 2 & 3 & 3 & -2 & 64 & $\mathrm{~B}>\mathrm{N}>\mathrm{Zn}>\mathrm{S}>\mathrm{P}>\mathrm{Cu}>\mathrm{Ca}=\mathrm{Fe}=\mathrm{Mn}>\mathrm{Mg}>\mathrm{K}$ \\
\hline 3 & -2 & 4 & 4 & 2 & -24 & -9 & 17 & 6 & 2 & 2 & -1 & 71 & $\mathrm{Mg}>\mathrm{S}>\mathrm{N}>\mathrm{Zn}>\mathrm{Ca}=\mathrm{Fe}=\mathrm{Mn}>\mathrm{P}=\mathrm{K}>\mathrm{Cu}>\mathrm{B}$ \\
\hline 4 & 1 & 7 & 16 & 0 & 6 & -4 & -24 & -1 & -3 & 8 & -6 & 77 & $\mathrm{~B}>\mathrm{Zn}>\mathrm{S}>\mathrm{Fe}>\mathrm{Cu}>\mathrm{Ca}>\mathrm{N}>\mathrm{Mg}>\mathrm{P}>\mathrm{Mn}>\mathrm{K}$ \\
\hline 5 & -7 & -2 & -3 & 0 & 4 & -2 & 18 & -1 & 5 & 3 & -16 & 61 & $\mathrm{Zn}>\mathrm{N}>\mathrm{K}>\mathrm{P}=\mathrm{S}>\mathrm{Cu}>\mathrm{Ca}>\mathrm{Mn}>\mathrm{Mg}>\mathrm{Fe}>\mathrm{B}$ \\
\hline 6 & -3 & 3 & -6 & -5 & 3 & -3 & 16 & 4 & -2 & 0 & -8 & 54 & $\mathrm{Zn}>\mathrm{K}>\mathrm{Ca}>\mathrm{N}=\mathrm{S}>\mathrm{Fe}>\mathrm{Mn}>\mathrm{P}=\mathrm{Mg}>\mathrm{Cu}>\mathrm{B}$ \\
\hline 7 & 5 & 0 & 19 & 0 & 1 & -6 & -19 & -3 & -4 & 7 & -3 & 69 & $\mathrm{~B}>\mathrm{S}>\mathrm{Fe}>\mathrm{Cu}=\mathrm{Zn}>\mathrm{P}=\mathrm{Ca}>\mathrm{Mg}>\mathrm{N}>\mathrm{Mn}>\mathrm{K}$ \\
\hline 8 & 0 & 6 & 15 & 0 & -1 & -2 & -24 & 2 & 4 & 6 & -6 & 67 & $\mathrm{~B}>\mathrm{Zn}>\mathrm{S}>\mathrm{Mg}>\mathrm{Ca}=\mathrm{N}>\mathrm{Cu}>\mathrm{Fe}>\mathrm{P}=\mathrm{Mn}>\mathrm{K}$ \\
\hline 9 & -6 & -1 & 15 & 2 & 4 & -3 & -23 & 5 & -4 & 9 & 0 & 73 & $\mathrm{~B}>\mathrm{N}>\mathrm{Fe}>\mathrm{S}>\mathrm{P}>\mathrm{Zn}>\mathrm{Ca}>\mathrm{Mg}>\mathrm{Cu}>\mathrm{Mn}>\mathrm{K}$ \\
\hline 10 & -5 & -5 & -3 & -2 & 7 & -5 & 15 & 1 & 3 & 2 & -8 & 56 & $\mathrm{Zn}>\mathrm{N}=\mathrm{P}=\mathrm{S}>\mathrm{K}>\mathrm{Ca}>\mathrm{Cu}>\mathrm{Mn}>\mathrm{Fe}>\mathrm{Mg}>\mathrm{B}$ \\
\hline 11 & 4 & 6 & 18 & 1 & -8 & -4 & -20 & 0 & 2 & 8 & -6 & 77 & $\mathrm{~B}>\mathrm{Mg}>\mathrm{Zn}>\mathrm{S}>\mathrm{Cu}>\mathrm{Ca}>\mathrm{Fe}>\mathrm{N}>\mathrm{P}>\mathrm{Mn}>\mathrm{K}$ \\
\hline 12 & -2 & -8 & 16 & 0 & 0 & 1 & -23 & 2 & 12 & 8 & -5 & 78 & $\mathrm{~B}>\mathrm{P}>\mathrm{Zn}>\mathrm{N}>\mathrm{Ca}=\mathrm{Mg}>\mathrm{S}>\mathrm{Cu}>\mathrm{Mn}>\mathrm{Fe}>\mathrm{K}$ \\
\hline 13 & 3 & 3 & 18 & 1 & -6 & -2 & -22 & 4 & 0 & 11 & -9 & 79 & $\mathrm{~B}>\mathrm{Zn}>\mathrm{Mg}>\mathrm{S}>\mathrm{Fe}>\mathrm{Ca}>\mathrm{N}=\mathrm{P}>\mathrm{Cu}>\mathrm{Mn}>\mathrm{K}$ \\
\hline 14 & -3 & -2 & 17 & 4 & 0 & -3 & -23 & 2 & 4 & 11 & -6 & 76 & $\mathrm{~B}>\mathrm{Zn}>\mathrm{N}=\mathrm{S}>\mathrm{P}>\mathrm{Mg}>\mathrm{Cu}>\mathrm{Ca}=\mathrm{Fe}>\mathrm{Mn}>\mathrm{K}$ \\
\hline 15 & -10 & -5 & 6 & 2 & -6 & -8 & 14 & 3 & -3 & 13 & -6 & 74 & $\mathrm{~N}>\mathrm{S}>\mathrm{Mg}>\mathrm{Zn}>\mathrm{P}>\mathrm{Fe}>\mathrm{Ca}>\mathrm{Cu}>\mathrm{K}>\mathrm{Mn}>\mathrm{B}$ \\
\hline 16 & -4 & 1 & 15 & 0 & 9 & 0 & -23 & -1 & 13 & 3 & -12 & 83 & $\mathrm{~B}>\mathrm{Zn}>\mathrm{N}>\mathrm{Cu}>\mathrm{Ca}=\mathrm{S}>\mathrm{P}>\mathrm{Mn}>\mathrm{Mg}>\mathrm{Fe}>\mathrm{K}$ \\
\hline 17 & -5 & -2 & 23 & -1 & 5 & -3 & -15 & 7 & 4 & -6 & -7 & 78 & $\mathrm{~B}>\mathrm{Zn}>\mathrm{Mn}>\mathrm{N}>\mathrm{S}>\mathrm{P}>\mathrm{Ca}>\mathrm{Fe}>\mathrm{Mg}>\mathrm{Cu}>\mathrm{K}$ \\
\hline 18 & -6 & -4 & 23 & 1 & 6 & -2 & -15 & 6 & 0 & -2 & -7 & 72 & $\mathrm{~B}>\mathrm{Zn}>\mathrm{N}>\mathrm{P}>\mathrm{S}=\mathrm{Mn}>\mathrm{Fe}>\mathrm{Ca}>\mathrm{Cu}=\mathrm{Mg}>\mathrm{K}$ \\
\hline 19 & -11 & -4 & -1 & -7 & 2 & -5 & 38 & 5 & -1 & -6 & -8 & 89 & $\mathrm{~N}>\mathrm{Zn}>\mathrm{Ca}>\mathrm{Mn}>\mathrm{S}>\mathrm{P}>\mathrm{K}=\mathrm{Fe}>\mathrm{Mg}>\mathrm{Cu}>\mathrm{B}$ \\
\hline 20 & -4 & -4 & 17 & -4 & 11 & 1 & -22 & 0 & 10 & 2 & -5 & 80 & $\mathrm{~B}>\mathrm{Zn}>\mathrm{N}=\mathrm{P}=\mathrm{Ca}>\mathrm{Cu}>\mathrm{S}>\mathrm{Mn}>\mathrm{Fe}>\mathrm{Mg}>\mathrm{K}$ \\
\hline 21 & -11 & -1 & 10 & -2 & 9 & -6 & 17 & -2 & 2 & -7 & -9 & 76 & $\mathrm{~N}>\mathrm{Zn}>\mathrm{Mn}>\mathrm{S}>\mathrm{Cu}=\mathrm{Ca}>\mathrm{P}>\mathrm{Fe}>\mathrm{Mg}>\mathrm{K}>\mathrm{B}$ \\
\hline 22 & -4 & 2 & 24 & -2 & 2 & -2 & -14 & 0 & -1 & 1 & -7 & 60 & $\mathrm{~B}>\mathrm{Zn}>\mathrm{N}>\mathrm{Ca}=\mathrm{S}>\mathrm{Fe}>\mathrm{Cu}>\mathrm{Mn}>\mathrm{P}=\mathrm{Mg}>\mathrm{K}$ \\
\hline 23 & -5 & -2 & -1 & -3 & 3 & -2 & 17 & 6 & 2 & -7 & -7 & 56 & $\mathrm{Zn}=\mathrm{Mn}>\mathrm{N}>\mathrm{Ca}>\mathrm{P}=\mathrm{S}>\mathrm{K}>\mathrm{Fe}>\mathrm{Mg}>\mathrm{Cu}>\mathrm{B}$ \\
\hline 24 & -2 & -2 & 20 & -3 & 1 & 1 & -18 & 7 & 8 & -3 & -7 & 71 & $\mathrm{~B}>\mathrm{Zn}>\mathrm{Mn}=\mathrm{Ca}>\mathrm{N}=\mathrm{P}>\mathrm{Mg}=\mathrm{S}>\mathrm{Cu}>\mathrm{Fe}>\mathrm{K}$ \\
\hline 25 & -6 & 0 & 20 & 0 & 12 & -3 & -18 & 3 & 4 & -2 & -10 & 79 & $\mathrm{~B}>\mathrm{Zn}>\mathrm{N}>\mathrm{S}>\mathrm{Mn}>\mathrm{P}=\mathrm{Ca}>\mathrm{Cu}>\mathrm{Fe}>\mathrm{Mg}>\mathrm{K}$ \\
\hline 26 & 0 & 3 & 10 & 5 & 2 & -1 & 25 & 8 & -2 & -46 & -4 & 107 & $\mathrm{Mn}>\mathrm{Zn}>\mathrm{Fe}>\mathrm{S}>\mathrm{N}>\mathrm{Mg}>\mathrm{P}>\mathrm{Ca}>\mathrm{Cu}>\mathrm{K}>\mathrm{B}$ \\
\hline 27 & 1 & -2 & 21 & 0 & 1 & -3 & -16 & 8 & -1 & -2 & -7 & 64 & $\mathrm{~B}>\mathrm{Zn}>\mathrm{S}>\mathrm{P}=\mathrm{Mn}>\mathrm{Fe}>\mathrm{Ca}>\mathrm{N}=\mathrm{Mg}>\mathrm{Cu}>\mathrm{K}$ \\
\hline 28 & -3 & -5 & 2 & -5 & -2 & -7 & 17 & 8 & 1 & 1 & -7 & 59 & $\mathrm{Zn}=\mathrm{S}>\mathrm{P}=\mathrm{Ca}>\mathrm{N}>\mathrm{Mg}>\mathrm{Fe}=\mathrm{Mn}>\mathrm{K}>\mathrm{Cu}>\mathrm{B}$ \\
\hline 29 & 0 & 5 & 25 & 9 & 24 & -1 & -15 & 10 & -5 & -47 & -6 & 146 & $\mathrm{Mn}>\mathrm{B}>\mathrm{Zn}>\mathrm{Fe}>\mathrm{S}>\mathrm{N}>\mathrm{P}>\mathrm{Ca}>\mathrm{Cu}>\mathrm{Mg}>\mathrm{K}$ \\
\hline 30 & -2 & -1 & 2 & 4 & 6 & -4 & 21 & 1 & -13 & -4 & -13 & 72 & $\mathrm{Zn}=\mathrm{Fe}>\mathrm{Mn}=\mathrm{S}>\mathrm{N}>\mathrm{P}>\mathrm{Cu}>\mathrm{K}>\mathrm{Ca}>\mathrm{Mg}>\mathrm{B}$ \\
\hline 31 & -9 & -4 & 6 & -1 & 3 & -5 & 16 & 5 & -2 & -6 & -2 & 60 & $\mathrm{~N}>\mathrm{Mn}>\mathrm{S}>\mathrm{P}>\mathrm{Fe}=\mathrm{Zn}>\mathrm{Ca}>\mathrm{Mg}>\mathrm{Cu}>\mathrm{K}>\mathrm{B}$ \\
\hline 32 & 1 & -1 & 21 & -3 & 2 & -5 & -16 & 9 & -13 & 2 & 0 & 72 & $\mathrm{~B}>\mathrm{Fe}>\mathrm{S}>\mathrm{Ca}>\mathrm{P}>\mathrm{Zn}>\mathrm{N}>\mathrm{Mg}=\mathrm{Mn}>\mathrm{Cu}>\mathrm{K}$ \\
\hline 33 & 1 & 7 & 19 & -4 & -6 & -1 & -19 & 8 & 6 & -4 & -6 & 81 & $\mathrm{~B}>\mathrm{Zn}=\mathrm{Mg}>\mathrm{Mn}=\mathrm{Ca}>\mathrm{S}>\mathrm{N}>\mathrm{Fe}>\mathrm{P}>\mathrm{Cu}>\mathrm{K}$ \\
\hline 34 & 2 & 0 & 10 & 4 & -16 & -17 & 26 & -2 & 5 & -9 & 0 & 91 & $\mathrm{~S}>\mathrm{Mg}>\mathrm{Mn}>\mathrm{Cu}>\mathrm{P}=\mathrm{Zn}>\mathrm{N}>\mathrm{Ca}>\mathrm{Fe}>\mathrm{K}>\mathrm{B}$ \\
\hline 35 & 1 & -1 & 16 & -1 & 10 & 0 & -23 & 0 & 0 & 2 & -6 & 62 & $\mathrm{~B}>\mathrm{Zn}>\mathrm{P}=\mathrm{Ca}>\mathrm{S}=\mathrm{Cu}=\mathrm{Fe}>\mathrm{N}>\mathrm{Mn}>\mathrm{Mg}>\mathrm{K}$ \\
\hline 36 & 4 & 0 & 16 & -4 & 9 & -1 & -21 & 7 & -1 & 2 & -10 & 75 & $\mathrm{~B}>\mathrm{Zn}>\mathrm{Ca}>\mathrm{S}=\mathrm{Fe}>\mathrm{P}>\mathrm{Mn}>\mathrm{N}>\mathrm{Cu}>\mathrm{Mg}>\mathrm{K}$ \\
\hline 37 & 4 & 2 & 16 & 1 & -2 & -1 & -22 & 7 & 3 & -3 & -5 & 65 & $\mathrm{~B}>\mathrm{Zn}>\mathrm{Mn}>\mathrm{Mg}>\mathrm{S}>\mathrm{Ca}>\mathrm{P}>\mathrm{Fe}>\mathrm{N}>\mathrm{Cu}>\mathrm{K}$ \\
\hline 38 & -2 & 2 & -1 & -2 & 3 & -3 & 17 & -1 & 0 & -1 & -12 & 45 & $\mathrm{Zn}>\mathrm{S}>\mathrm{N}=\mathrm{Ca}>\mathrm{K}=\mathrm{Cu}=\mathrm{Mn}>\mathrm{Fe}>\mathrm{P}>\mathrm{Mg}>\mathrm{B}$ \\
\hline 39 & 3 & 1 & 17 & -3 & 10 & -5 & -20 & 8 & -4 & -4 & -2 & 78 & $\mathrm{~B}>\mathrm{S}>\mathrm{Mn}=\mathrm{Fe}>\mathrm{Ca}>\mathrm{Zn}>\mathrm{P}>\mathrm{N}>\mathrm{Cu}>\mathrm{Mg}>\mathrm{K}$ \\
\hline 40 & 5 & 1 & 17 & 1 & 4 & -3 & -21 & 5 & 3 & -3 & -8 & 72 & $\mathrm{~B}>\mathrm{Zn}>\mathrm{Mn}=\mathrm{S}>\mathrm{P}=\mathrm{Ca}>\mathrm{Fe}>\mathrm{Mg}>\mathrm{N}=\mathrm{Cu}>\mathrm{K}$ \\
\hline 41 & 0 & 5 & 16 & -1 & 10 & -2 & -23 & 0 & -5 & 3 & -4 & 69 & $\mathrm{~B}>\mathrm{Fe}>\mathrm{Zn}>\mathrm{S}>\mathrm{Ca}>\mathrm{N}=\mathrm{Cu}>\mathrm{Mn}>\mathrm{P}>\mathrm{Mg}>\mathrm{K}$ \\
\hline 42 & 2 & 4 & 17 & 4 & 1 & -4 & -25 & 10 & -3 & -3 & -2 & 74 & $\mathrm{~B}>\mathrm{S}>\mathrm{Fe}=\mathrm{Mn}>\mathrm{Zn}>\mathrm{Mg}>\mathrm{N}>\mathrm{P}=\mathrm{Ca}>\mathrm{Cu}>\mathrm{K}$ \\
\hline 43 & 2 & 8 & 16 & -6 & 0 & -3 & -25 & 6 & -1 & 3 & 0 & 69 & $\mathrm{~B}>\mathrm{Ca}>\mathrm{S}>\mathrm{Fe}>\mathrm{Mg}=\mathrm{Zn}>\mathrm{N}>\mathrm{Mn}>\mathrm{Cu}>\mathrm{P}>\mathrm{K}$ \\
\hline 44 & 7 & -3 & 17 & -4 & 12 & -4 & -23 & 1 & 5 & 2 & -10 & 87 & $\mathrm{~B}>\mathrm{Zn}>\mathrm{S}=\mathrm{Ca}>\mathrm{P}>\mathrm{Cu}>\mathrm{Mn}>\mathrm{Fe}>\mathrm{N}>\mathrm{Mg}>\mathrm{K}$ \\
\hline 45 & -2 & 4 & -13 & 1 & 4 & -6 & 17 & 0 & -11 & 0 & 4 & 64 & $\mathrm{~K}>\mathrm{Fe}>\mathrm{S}>\mathrm{N}>\mathrm{Mn}=\mathrm{Cu}>\mathrm{Ca}>\mathrm{P}=\mathrm{Zn}=\mathrm{Mg}>\mathrm{B}$ \\
\hline 46 & 0 & 1 & 16 & 2 & 12 & -3 & -24 & 1 & 2 & -2 & -4 & 69 & $\mathrm{~B}>\mathrm{Zn}>\mathrm{S}>\mathrm{Mn}>\mathrm{N}>\mathrm{P}=\mathrm{Cu}>\mathrm{Ca}=\mathrm{Fe}>\mathrm{Mg}>\mathrm{K}$ \\
\hline 47 & -1 & 2 & -4 & -5 & 4 & -7 & 18 & 5 & 0 & -3 & -9 & 57 & $\mathrm{Zn}>\mathrm{S}>\mathrm{Ca}>\mathrm{K}>\mathrm{Mn}>\mathrm{N}>\mathrm{Fe}>\mathrm{P}>\mathrm{Mg}>\mathrm{Cu}>\mathrm{B}$ \\
\hline 48 & 1 & 0 & 15 & -6 & 10 & -3 & -24 & 8 & 7 & -1 & -6 & 82 & $\mathrm{~B}>\mathrm{Zn}=\mathrm{Ca}>\mathrm{S}>\mathrm{Mn}>\mathrm{P}>\mathrm{N}>\mathrm{Fe}>\mathrm{Cu}>\mathrm{Mg}>\mathrm{K}$ \\
\hline
\end{tabular}

${ }^{1}$ Número da amostra. ${ }^{2}$ Utilizou-se, para cálculo do IBN, o procedimento de Beaufils (1973). 
Tabela 5. Percentagem de ocorrência dos nutrientes mais limitantes por deficiência, diagnosticados pelos índices DRIS obtidos pela análise foliar, para a cultura do algodão, em 48 amostras (Silvânia, GO, safra 2007/2008).

\begin{tabular}{lrrrrr}
\hline \multirow{2}{*}{ Variável } & \multicolumn{4}{c}{$\%$ de ocorrência ${ }^{1}$} & $\begin{array}{c}\text { Diferença em relação às faixas de } \\
\text { concentração }(\%)^{2}\end{array}$ \\
\cline { 2 - 4 } & Total & $1^{\mathrm{a}}$ ordem & $2^{\mathrm{a}}$ ordem & $3^{\mathrm{a}}$ ordem & 52,58 \\
$\mathrm{~N}$ & 9,92 & 8,33 & 10,42 & 20,83 & $-8,33$ \\
$\mathrm{P}$ & 8,33 & 0,00 & 6,25 & 4,17 & 94,73 \\
$\mathrm{~K}$ & 3,17 & 2,08 & 2,08 & 4,17 & 20,47 \\
$\mathrm{Ca}$ & 8,73 & 0,00 & 6,25 & 20,83 & 0,63 \\
$\mathrm{Mg}$ & 3,57 & 2,08 & 6,25 & 4,17 & 82,94 \\
$\mathrm{~S}$ & 17,06 & 4,17 & 18,75 & 27,08 & 54,00 \\
$\mathrm{~B}$ & 12,70 & 64,58 & 2,08 & 0,00 & $-2,78$ \\
$\mathrm{Cu}$ & 2,78 & 0,00 & 0,00 & 0,00 & $-7,94$ \\
$\mathrm{Fe}$ & 7,94 & 2,08 & 8,33 & 10,42 & $-4,53$ \\
$\mathrm{Mn}$ & 8,73 & 6,25 & 4,17 & 18,75 & 80,84 \\
$\mathrm{Zn}$ & 17,06 & 16,67 & 47,92 & 10,42 & \\
\hline
\end{tabular}

${ }^{1} \mathrm{Na}$ ocorrência total, consideram-se todos os índices negativos. Nas percentagens de ocorrência de $1^{\mathrm{a}}, 2^{\mathrm{a}}$ e $3^{\mathrm{a}}$ ordem, consideram-se os primeiros, segundos e terceiros índices mais negativos de cada amostra, respectivamente. ${ }^{2}$ Diferença entre percentagem de ocorrência de amostras na classe abaixo da adequada, pelo método das Faixas de Concentração proposto por Malavolta (2006), e a percentagem de ocorrência total, pelo método DRIS.

ordens mais negativas de limitação. As faixas de equilíbrio nutricional podem apresentar variações, de acordo com a sensibilidade com que os diferentes autores obtiveram seus bancos de dados, incluindo variedades e regiões diferentes. Para Kelling \& Schulte (1986), a faixa de melhor balanço nutricional situa-se no intervalo de -15 a +15 , enquanto, para Escano et al. (1981), está entre $-1,0$ e $+1,5$, e, para Soltanpour et al. (1995), de $-7 \mathrm{a}+7$.

Verificou-se, também, que os melhores IBNs (valores mais próximos de zero) foram encontrados nas amostras de número $2 ; 5 ; 6 ; 10 ; 22 ; 23 ; 27 ; 28$; $31 ; 35 ; 37 ; 38 ; 45 ;$ e 47 (Tabela 4), já que as mesmas apresentaram plantas com melhor balanço nutricional.

Ao se compararem as percentagens de ocorrência de limitação total dos nutrientes (Tabela 5) com as distribuições de frequência das faixas de concentração (Tabela 3), verifica-se que os métodos de diagnose do estado nutricional apresentaram alguns resultados discordantes, como por exemplo, para o $\mathrm{K}$ e para os micronutrientes $\mathrm{Fe}, \mathrm{Mn}$ e $\mathrm{Cu}$.

Pelo método de diagnose DRIS, a ordem de limitação, a partir da percentagem de ocorrência de limitação total, foi $\mathrm{S}=\mathrm{Zn}>\mathrm{B}>\mathrm{N}>\mathrm{Ca}=\mathrm{Mn}>$ $\mathrm{P}>\mathrm{Fe}>\mathrm{Mg}>\mathrm{K}>\mathrm{Cu}$. Verifica-se que as maiores diferenças entre os métodos referem-se às variáveis K (94,73\%), S (82,94\%), Zn (80,84\%), B (54,00\%) e N (52,58\%) (Tabela 5).

Para o K, o método DRIS apresentou 94,73\% de deficiências a menos que o método das Faixas de Concentração (Tabela 5), demonstrando que, realmente, este não é o mais limitante, pelo DRIS. Analisando-se a Tabela 4, observa-se que, aproxima- damente, $66 \%$ das amostras de folha apresentaram $\mathrm{K}$ na última ordem de limitação (os valores mais positivos de cada ponto de amostragem), evidenciando um possível excesso do nutriente.

Em relação ao $\mathrm{P}, \mathrm{Fe}, \mathrm{Mn}$ e $\mathrm{Cu}$, o método DRIS apresentou $8,33 \% ; 7,94 \% ; 4,53 \%$; e $2,78 \%$ de amostras com deficiência deste nutriente, respectivamente (Tabela 5), enquanto o método das Faixas não apresentou amostras deficientes em $\mathrm{Fe} e$, ainda, classificou a maioria das amostras como acima da faixa adequada para $\mathrm{P}, \mathrm{Mn}$ e $\mathrm{Cu}$ (Tabela 3 ).

Como já discutido anteriormente, as faixas propostas por Malavolta (2006) são muito estreitas para micronutrientes e, em se tratando de cultivares modernas, como a Fibermax 966 e 993, que são responsivas a solos bem corrigidos e com alta fertilidade, pode ser que esta interpretação, segundo Malavolta (2006), não seja adequada.

O método das Faixas de Concentração apresentou maior quantidade de amostras deficientes em $\mathrm{N}, \mathrm{B}, \mathrm{S}$ e $\mathrm{Zn}$ do que o método DRIS, aproximadamente $52,58 \% ; 54,00 \% ; 82,94 \%$; e $80,84 \%$, respectivamente, a mais de amostras deficientes, levando-se em consideração a percentagem de ocorrência total do método DRIS. Entretanto, para estes nutrientes, o DRIS apresentou uma grande percentagem de amostras limitantes em primeira e segunda ordem, especialmente o B (Tabela 5).

Este resultado tem grande expressão no diagnóstico dos nutrientes mais limitantes à produção e supre a carência dos métodos tradicionais de interpretação, que não conseguem detectar qual é o nutriente mais limitante, quando mais de dois 
nutrientes encontram-se deficientes na amostra (Hanson 1981). Portanto, para os dois métodos de interpretação, grande parte das amostras apresentou, como nutrientes mais limitantes por deficiência, N, $\mathrm{S}, \mathrm{B}$ e $\mathrm{Zn}$. O método DRIS ainda apresentou maior sensibilidade para diagnosticar deficiências de micronutrientes, especialmente para B, Fe e Mn. Serra et al. (2010b) também diagnosticaram que o Mn foi o elemento que se apresentou com maior frequência nos talhões, como limitante por falta.

$\mathrm{O} \mathrm{N}$ é o nutriente extraído do solo em maior quantidade pelo algodoeiro. Sabe-se que, na área avaliada, foram feitas duas aplicações de $125 \mathrm{~kg} \mathrm{ha}^{-1}$ de ureia em cobertura e, mesmo assim, foi observada deficiência de $\mathrm{N}$ nas folhas. Este fato deve ser consequência de problemas com a lixiviação e volatilização do nutriente, em decorrência da forma de aplicação empregada (a lanço). Segundo Sabino et al. (1994), sob condições tropicais, o N é, possivelmente, o fator que mais limita a produtividade, devido às grandes perdas por lixiviação e/ou volatilização.

Outros autores também confirmaram que o $\mathrm{N}$ está diretamente ligado à produtividade, por exemplo, Medeiros et al. (2001) constataram que o maior peso de capulho de algodão foi obtido quando foram aplicadas as doses de $50-150 \mathrm{~kg}$ de $\mathrm{N} \mathrm{ha}^{-1}$. Já Silva et al. (2001) verificaram que doses crescentes de $\mathrm{N}\left(0 \mathrm{~kg} \mathrm{ha}^{-1}, 60 \mathrm{~kg} \mathrm{ha}^{-1}, 120 \mathrm{~kg} \mathrm{ha}^{-1}\right.$ e $\left.180 \mathrm{~kg} \mathrm{ha}^{-1}\right)$ promoveram aumento linear da massa dos capulhos.

As limitações de S na área podem estar relacionadas com as deficiências naturais de enxofre, nos solos de Cerrado, que, geralmente, estão associadas à queima frequente destas áreas (Kamprath 1973); ao cultivo intensivo por vários anos, com oxidação da matéria orgânica do solo; e à utilização constante de fertilizantes concentrados à base de NPK, que não contenham S em sua composição (Lopes 1984).

Dentre os micronutrientes exigidos pela cultura do algodão, o B é um dos mais responsivos. Foram realizadas adubações foliares com B, mas sua deficiência pode ter resultado de uma calagem excessiva ou mal feita. Segundo Staut \& Kurihara (2001), a deficiência de B aumenta na presença de calagem excessiva, ou em solos pobres em matéria orgânica, principalmente em regiões sujeitas a períodos de chuvas intensas e temperaturas elevadas, onde este nutriente pode ser perdido por lixiviação, por apresentar grande mobilidade no solo (Malavolta 2006, Communar \& Keren 2007, Rosolem \& Bíscaro 2007).
Apesar de a área em estudo adotar um sistema de preparo do solo mais conservacionista (plantio semidireto), verificou-se que alguns pontos de amostragem na área apresentaram elevada erosão e isto pode ter contribuído para a diminuição da matéria orgânica e deficiência de B.

Outro micronutriente considerado limitante por deficiência foi o Zn. Este resultado corrobora os dados de Lopes (1984), o qual observou que a maioria dos solos (79\%) sob Cerrado apresenta entre $0,5 \mathrm{mg} \mathrm{dm}^{-3} \mathrm{e} 0,8 \mathrm{mg} \mathrm{dm}^{-3} \mathrm{de} \mathrm{Zn}$. As condições de solo que provocam a deficiência deste micronutriente são o baixo teor de Zn e matéria orgânica no solo, calagem excessiva ou mal feita e excessos de N, P, Ca, Mg, Cu, Fe e metais pesados (Malavolta \& Kliemann 1985).

Segundo Malavolta \& Gorostiaga (1974), a calagem e a adubação fosfatada podem provocar deficiências de $\mathrm{Zn}$, por meio da diminuição da absorção do elemento provocada pelo $\mathrm{P}$ (inibição não competitiva) e insolubilização do Zn pelo calcário.

Foi realizada, na área em estudo, uma calagem superficial, 2 meses antes do plantio, e uma adubação de plantio e adubação foliar com fósforo. Então, uma possível causa da deficiência de Zn observada na análise foliar pode ser a calagem e a dose de $\mathrm{P}$ empregada, que limitaram a disponibilidade de $\mathrm{Zn}$ por insolubilização.

\section{CONCLUSÕES}

1. Os nutrientes N, S, B e Zn, obtidos pelas análises foliares e diagnosticados pelo método DRIS e Faixas de Concentração, são os mais limitantes para a cultura do algodão, na área comercial estudada.

2. O método DRIS apresentou maior sensibilidade para diagnosticar deficiências de micronutrientes, especialmente B, Fe e Zn.

3. Por meio do DRIS, é possível estabelecer a ordem de limitação dos nutrientes, nas análises foliares.

4. Os dois métodos de interpretação dos resultados de análise foliar podem ser utilizados de forma complementar e isto é importante para permitir a elevação da produtividade de talhões de lavouras de algodão com nutrição mineral equilibrada.

\section{REFERÊNCIAS}

ALVAREZ, V. H.; LEITE, R. A. Fundamentos estatísticos das fórmulas usadas para cálculo dos índices dos nutrientes no Sistema Integrado de Diagnose e Recomendação - 
DRIS. In: REUNIÃO BRASILEIRA DE FERTILIDADE DO SOLO E NUTRIÇÃO DE PLANTAS, 20., 1992, Piracicaba. Anais... Piracicaba: SBCS, 1992. p. 186-187.

ANUÁRIO BRASILEIRO DO ALGODÃO. Santa Cruz do Sul: Gazeta, 2010. Disponível em: <http://www. anuarios.com.br/>. Acesso em: 15 jan. 2011.

BALDOCK, J. O.; SCHULTE, E. E. Plant analysis with standardized scores combines DRIS and sufficiency range approaches for corn. Agronomy Journal, Madison, v. 88, n. 3, p. 448-456, 1996.

BATAGLiA, O. C. et al. Análise química de plantas. Campinas: Instituto Agronômico, 1978. (Boletim técnico, 87).

BEAUFILS, E. R. Diagnosis and recommendation integrated system (DRIS): a general scheme for experimentation and calibration based on principles developed from research in plant nutrition. Soil Science Bulletin, Pietermaritzburg, v. 1, n. 1, p. 1-132, 1973.

CHAPMANN, H. D. Diagnosis criteria for plants and soils. Riverside: University of California, 1973.

COMMUNAR, G.; KEREN, R. Effect of transient irrigation on boron transport in soils. Soil Science Society of America Journal, Madison, v. 71, n. 2, p. 306-313, 2007.

COMPANHIA NACIONAL DE ABASTECIMENTO (Conab). Acompanhamento de safra brasileira 2010/2011. 2011. Disponível em: $<\mathrm{http}: / / \mathrm{www}$. conab.gov.br/>. Acesso em: 15 jan. 2011.

DARA, S. T.; FIXEN, P. E.; GELDERMAN, R. H. Sufficiency level and diagnosis and recommendation integrated system approaches for evaluating the nitrogen status of the corn. Agronomy Journal, Madison, v. 84, n. 6, p. 1006-1010, 1992.

EMPRESA BRASILEIRA DE PESQUISA AGROPECUÁRIA (Embrapa). Centro Nacional de Pesquisa de Solos. Sistema brasileiro de classificação de solos. Brasília, DF: Embrapa Produção da Informação; Rio de Janeiro: Embrapa Solos, 1999.

ESCANO, C. R.; JONES, C. A.; UEHARA, G. Nutrient diagnosis in corn on hydric dystrandepts: II. Comparison of two systems of tissue diagnosis. Soil Science Society of America Journal, Madison, v. 45, n. 3, p. 1140-1144, 1981.

FREUND, R. J.; LITTELL, R. C. SAS for linear models: a guide to the ANOVA and GLM procedures. Cary: SAS Institute, 1981.

HANSON, R. G. DRIS evaluation of N, P, K status of determinant soybean in Brazil. Communications in Soil Science and Plant Analysis, New York, v. 12, n. 9, p. 933948, 1981.

KAMPRATH, E. J. Sufur. In: SANCHEZ, P. A. (Ed.). $A$ review of soils research in tropical Latim America.
Raleigh: North Carolina Agricultural Experiment Station, 1973. p. 179-180. (Technical bulletin, 219).

KELLING, K. A.; SCHULTE, E. E. DRIS as a part of a routine plant analysis program. Journal Fertility, Manchester, v. 3, n. 3, p. 107-112, 1986.

KURIHARA, C. H.; STAUT, L. A.; MAEDA, S. Faixas de suficiência para teores foliares de nutrientes em algodão e soja, definidas pelo uso do método DRIS de diagnose do estado nutricional. In: REUNIÃO BRASILEIRA DE FERTILIDADE DO SOLO E NUTRIÇÃO DE PLANTAS, 28., 2008, Londrina. Anais... Londrina: Embrapa Soja/ SBCS/IAPAR/UEL, 2008. 1 CD-ROM.

LEANDRO, W. M. Sistema integrado de diagnose e recomendação (DRIS) para a cultura da soja (Glycine max L. Merril) na região de Rio Verde-GO. 1998. 157 f. Tese (Doutorado em Agronomia: Produção Vegetal)-Escola de Agronomia e Engenharia de Alimentos, Universidade Federal de Goiás, Goiânia, 1998.

LOPES, A. S. Solos sob Cerrado: características, propriedades e manejo. 2. ed. Piracicaba: Potafos, 1984.

MALAVOLTA, E. Manual de nutrição mineral de plantas. São Paulo: Agronômica Ceres, 2006.

MALAVOlTA, E.; GOROSTIAGA, O. L. Studies on the zinc phosphate relationships in plants. In: INTERNATIONAL COLLOQUIUM ON PLANT ANALYSIS AND FERTILIZER PROBLEMS, 7., 1974, Hanover. Proceedings... Hanover: German Society of plant Nutrition, 1974. p. 261-272.

Malavolta, E.; KLIEMAnN, H. J. Desordens nutricionais no Cerrado. Piracicaba: Potafos, 1985.

MALAVOLTA, E.; VITTI, G. C.; OLIVEIRA, S. A. Avaliação do estado nutricional das plantas: princípios e aplicações. 2. ed. Piracicaba: Potafos, 1997.

MEDEIROS, J. C. et al. Safra 1999/2000: principais ações de pesquisa e transferência de tecnologia para o algodoeiro no Estado de Goiás. Campina Grande: Embrapa/CNPA, 2001.

MORAIS, N. R. Critério de interpretação da qualidade do solo para a cotonicultura no Cerrado goiano. 2008. 79 f. Dissertação (Mestrado em Agronomia: Solo e Água)-Escola de Agronomia e Engenharia de Alimentos, Universidade Federal de Goiás, Goiânia, 2008.

REIS JÚNIOR, R. A.; MONNERAT, P. H. Norms establishment of the diagnosis and recommendation integrated system (DRIS) for nutritional diagnosis of sugarcane. Pesquisa Agropecuária Brasileira, Brasília, DF, v. 38, n. 2, p. 277-282, 2003.

ROSOLEM, C. A.; BÍSCARO, T. Adsorção e lixiviação de boro em Latossolo Vermelho-Amarelo. Pesquisa Agropecuária Brasileira, Brasília, DF, v. 42, n. 10, p. 14731478, 2007. 
SABINO, P. N. et al. Efeitos da aplicação de ureia e de sulfato de amônio nas características agronômicas e propriedades tecnológicas da fibra do algodoeiro. Bragantia, Campinas, v. 53, n. 1, p. 75-82, 1994.

SERRA, A. P. et al. Determinação de faixas normais de nutrientes no algodoeiro pelos métodos ChM, CND e DRIS. Revista Brasileira de Ciência do Solo, Viçosa, v. 34, n. 1, p. 105-113, 2010a.

SERRA, A. P. et al. Desenvolvimento de normas DRIS e CND e avaliação do estado nutricional da cultura do algodoeiro. Revista Brasileira de Ciência do Solo, Viçosa, v. 34, n. 1, p. 97-104, 2010 b.

SiLVA, M. A. C.; NATALE, W.; MALHEIROS, E. B. Estabelecimento de normas DRIS para a cultura do algodoeiro. In: CONGRESSO BRASILEIRO DE ALGODÃO, 7., 2007, Uberlândia. Anais... Brasília, DF: Embrapa, 2007. 1 CD-ROM.

SILVA, N. M. da et al. Seja o doutor do seu algodoeiro. Piracicaba: Potafos, 1995. (Arquivo do agrônomo, 8).

SOLTANPOUR, P. N.; MALAKOWTI, M. J.; RONAGHI, A. Comparison of diagnosis and recommendation system and nutrient sufficiency range for corn. Soil Science Society of America Journal, Madison, v. 59, n. 1, p. 133-139, 1995.

SOUZA, R. F. Sistema integrado de diagnose e recomendação (DRIS) para a cultura do algodão no município de Silvânia-Goiás. 2009. 116 f. Dissertação (Mestrado em Agronomia: Produção Vegetal)-Escola de Agronomia e Engenharia de Alimentos, Universidade Federal de Goiás, Goiânia, 2009.
STAUT, L. A.; KURIHARA, C. H. Calagem e adubação. In: EMBRAPA AGROPECUÁRIA OESTE. Algodão: tecnologias de produção. Dourados: Embrapa Agropecuária Oeste, 2001. p. 103-123.

SUMNER, M. E. Effect of corn leaf sampled on N, $\mathrm{P}, \mathrm{K}, \mathrm{Ca}$ and $\mathrm{Mg}$ content and calculated DRIS Index. Communications in Soil Science and Plant Analysis, New York, v. 8, n. 3, p. 269-280, 1977.

VALDAMERI, M. L; POUYÚ-ROJAS, E. Sistema integrado de diagnose e recomendação (DRIS) para a diagnose nutricional do algodoeiro em algumas áreas no Estado de Mato Grosso. In: REUNIÃO BRASILEIRA DE FERTILIDADE DO SOLO E NUTRIÇÃO DE PLANTAS, 28., 2008, Londrina. Anais... Londrina: Embrapa Soja/SBCS/IAPAR/UEL, 2008. 1 CD-ROM.

WALSH, L. M.; BEATON, J. D. Soil testing and plant analysis. Madison: Soil Science Society of America, 1973.

WALWORTH, J. L.; SUMNER, M. E. The diagnosis and recommendation system (DRIS). Advances in Soil Science, Boca Raton, v. 6, n. 1, p. 149-188, 1987. 